\title{
COUPLED PARABOLIC EQUATIONS FOR WAVE PROPAGATION *
}

\author{
KAI HUANG, KNUT SOLNA AND HONGKAI ZHAO ${ }^{\dagger}$
}

Dedicated to Professor George Papanicolaou on the occasion of his 60th birthday

\begin{abstract}
We develop an algorithm using two coupled parabolic equations for numerical simulation of wave propagation over long distances. The coupled parabolic equations are derived from a two mode wave decomposition. An iterative procedure is used in our numerical algorithm. The coupling between the two parabolic equations allow us to deal with fine scale inhomogeneities in the medium and capture the back scattering that are usually neglected in a one way parabolic approximation. We study stability issues of our numerical algorithm and present numerical examples.
\end{abstract}

Key words. parabolic approximation, Helmholtz equation, wave propagation

AMS subject classifications. $65,86-08$

1. Introduction. Efficient numerical algorithms are important in order to understand wave propagation in complex media. Resolving the wavelength is one of the basic sampling conditions for numerical algorithms. However, in many applications, such as underwater acoustics, communications and remote sensing, the wave propagates over a long distance which may be several order of magnitudes compared to the wavelength. If the medium is inhomogeneous there is an additional characteristic scale corresponding to the correlation length of the medium inhomogeneities. Wave propagation in a heterogeneous medium is thus a multi-scale problem in space and time, which poses a great challenge for numerical simulations. Here we focus on the simulation of time harmonic waves, that is, we reduce the full wave equation in space and time to the Helmholtz equation in space only by Fourier transformation in time. The Helmholtz equation gives a boundary value problem and it is very expensive to solve with a computational domain that is large compared to the wavelength. If the wave propagates in a weakly inhomogeneous medium, e.g., underwater acoustics or electromagnetic waves in the atmosphere $[8,15,19]$ the Helmholtz equation can be further simplified. In a situation where the propagation distance is much larger than the transverse dimension corresponding to a narrow angle geometry and when the back scattering can be neglected the Helmholtz equation can be reduced to a parabolic equation. The main advantage of the parabolic approximation is that it gives an initial value problem which is much easier to analyze and much cheaper to compute. The parabolic equation (PE) approximation provides an important tool for analysis and computation of wave propagation. More advanced parabolic approximations have been developed to deal with back scattering and wide angle geometries.

Marten de Hoop and coauthors have developed [5, 18] and analyzed a directional wave decomposition framework that involves generalized Bremmer series. They introduce a principal direction of propagation and decompose the acoustic wave equations into a coupled system of one way wave equations which leads to the Bremmer series approximation for the wave field via a high frequency series expansion. The $n$ 'th term in the series can be interpreted as the wave components being scattered $n$ times.

\footnotetext{
* Received August 15, 2004; accepted for publication November 19, 2004. The research is partially supported by ONR Grant N00014-02-1-0090, DARPA Grant N00014-02-1-0603 and NSF Grant 0307011.

$\dagger^{\dagger}$ Department of Mathematics, University of California at Irvine, CA 92697-3875, U.S.A. (khuang@math.uci.edu; ksolna@math.uci.edu; zhao@math.uci.edu).
} 
Various approaches have been proposed for approximating the Bremmer series with a paraxial type approach being presented in [18]. Here the coupled system of one way equations are approximated via a sparse approximation of the propagator over thin sections where rational approximation of the exponent provides numerical stability. In this procedure the different terms in the Bremmer series is then approximated by forward and backward sweeps. The approach has applications to for instance inverse scattering problems in seismics.

In [9] Fishman developed a one-way propagation formulation based on a wave-field decomposition and the a scattering operator approximation. The wave field is decomposed as $u=u^{(+)}+u^{(-)}$and the condition $u_{x}=i B(x)\left[u^{+}-u^{(-)}\right]$is required, where $x$ is propagation direction and $B(x)=\sqrt{\partial_{z}^{2}+k^{2}(x, z)}$ is the square root operator which can be identified in terms of the eigenvalues and eigenfunctions of the transverse operator $\partial_{z}^{2}+k^{2}$. The construction of several explicit, uniform asymptotic approximations of the square root Helmholtz operator are given in [10]. A re-formulation based on the Dirichlet-to-Neumann (DtN) map has also been derived in [12] and [13, 14]. An operator Riccati equation should be solved for decreasing propagating distance with an initial condition that matches the exact radiation condition at $\infty$. While the field should be solved for increasing propagating distance. This is practical only if the total number of operator equation solutions required is not very large.

A two way parabolic approximation motivated by underwater acoustics is introduced by Collins in [4]. In this paper the authors consider a generalization of the one way or outgoing parabolic approximation to handle medium variations in the range, that is, the propagation direction. Their approximation is motivated by applications to ocean wave-guides where often the depth variation of the medium parameters is stronger than the variation in range. The range-dependent medium is approximated by a sequence of range-independent sections. At each interface the reflected field is approximated via an iterative technique using the parabolic wave operator. The reflected field components are then propagated back via the incoming parabolic approximation. Here our focus is rather on relatively rapidly fluctuating small scale scatterers and we do not decompose the medium into range independent sections, thus the parabolic modes couple at all depths, moreover, we carry out many iterations in range.

We start with the Helmholtz equation and use a wave decomposition as in $[16,17]$ to derive a system of coupled parabolic equations. We use a bidirectional wave decomposition and get two coupled parabolic equations. Our decomposition of the wave field is based on local condition and can deal with fine local structure in the medium and capture high frequency components of the wavefield. The coupling comes from backscattering and transverse spreading of the wave. Based on this decomposition, a straightforward Jacobi type of iterative algorithm can be applied. However, this iterative algorithm is not stable and will not converge due to the lagged coupling in the iterative procedure. In this paper we modify these two equations and develop an efficient algorithm that is as simple as the standard PE approximation but can better deal with heterogeneities and back scattering.

We consider the Helmholtz equation in 2-dimensions for simplicity. However the algorithm can easily be generalized to the 3 -dimensional case. Numerical experiments are used to compare our method with the standard parabolic approximation.

The outline of the paper is as follows, in Section 2 we give a brief review of the standard parabolic approximation and the two way parabolic approximation. The coupled parabolic system and the absorbing boundary condition will be derived in Sections 3- 5. We present our numerical algorithm and numerical results in Section 
6. In the appendix we present a stability analysis.

2. The Parabolic Approximation. We consider the problem of propagation of acoustic signals over large distances. Let $\mathbf{u}(\mathbf{x}, t)$ and $p(\mathbf{x}, t)$ be the acoustic velocity and pressure satisfying the equation of continuity of momentum and mass

$$
\begin{array}{r}
\rho \mathbf{u}_{t}+\nabla p=\mathbf{F}(\mathbf{x}, z, t), \\
K^{-1} p_{t}+\nabla \cdot \mathbf{u}=0,
\end{array}
$$

where $t$ is time, $z$ is depth into the medium and defined so as to increase with depth, $(\mathbf{x}, z)=(x, y, z)$ are the space coordinates, $\rho$ is the density, $K$ is the bulk modulus and the source $\mathbf{F}(\mathbf{x}, \mathrm{t})$ is supported in the half-space $z<0$. We model the medium by $\rho=\rho_{0}$ constant and

$$
K^{-1}(\mathbf{x}, z)=\left\{\begin{array}{lr}
K_{0}^{-1} & z \in(-\infty, 0] \\
K_{p}^{-1}(\mathbf{x}, z)(1+\nu(\mathbf{x}, z)) & z \in(0, \infty)
\end{array}\right.
$$

The function $\nu$ modulating the compliance corresponds the medium fluctuations. In the case of a stationary random medium it is a zero-mean, stationary stochastic process whose statistics take on particular forms depending on the assumptions about the medium, whether it is locally layered, strongly or weakly heterogeneous media and so on [1].

At this stage, the source term is omitted, but it will be taken into account through the initial conditions for the parabolic equation.

Eliminating $\mathbf{u}$ from equations (1) and (2), we get

$$
\triangle p-\frac{\rho}{K} p_{t t}=0 .
$$

The time-harmonic version of (3) is the Helmholtz Equation:

$$
\triangle \hat{p}+(1+\nu) \omega^{2} \gamma^{2} \hat{p}=0
$$

where

$$
\gamma(\mathbf{x}, z)=\sqrt{K_{p}^{-1}(\mathbf{x}, z) \rho}
$$

and $\hat{p}$ is the Fourier transform of $p$ with respect to time:

$$
\hat{p}=\int p e^{i \omega t} d t .
$$

Note that below we suppress the 'hat' and that $\gamma$ represents an effective slowness.

The Helmholtz equation is associated with some specific boundary values. If the computational domain of this boundary value problem is large compared to the wavelength, solving the discretized linear system using a direct method may be impossible due to memory constraints. Moreover, since the linear system is not positive definite, usual iterative methods typically converge slowly, if at all. The parabolic approximation can be used to deal with this problem in various settings. The main idea is to neglect the back scattering and only consider forward going waves. The PE approximation becomes an initial value problem which significantly reduces the complexity for both analysis and computation. This approximation is accurate in many scenarios such as in range dependent ocean wave-guides or in the case of atmospheric wave propagation. 
We are interested in wave propagation mainly in one direction, along the $z$ axis in our notation. We use the plane wave ansatz

$$
p(\mathbf{x}, z)=A(\mathbf{x}, z) \exp \left(i k_{0} z\right),
$$

for the solution of Helmholtz Equation:

$$
\triangle p+\omega^{2} \gamma^{2}(\mathbf{x}, z)(1+\nu) p=0
$$

where $k_{0}$ is a reference wave number. The factor $\exp \left(i k_{0} z\right)$ in $(5)$ represents a plane wave traveling in the positive $z$ direction and is supposed to take out the rapid oscillations of $p$ in the $z$ direction; the function $A(\mathbf{x}, z)$ captures the modulation of the plane wave phase and usually varies slowly with $z$ in the context of the PE approximation.

Substitution of Equation (5) into Equation (6) gives

$$
\frac{\partial^{2} A}{\partial z^{2}}+2 i k_{0} \frac{\partial A}{\partial z}+\triangle_{\perp} A+\left[\omega^{2} \gamma^{2}(1+\nu)-k_{0}^{2}\right] A=0,
$$

with $\triangle_{\perp}$ being the Laplacian in the lateral coordinates $\mathbf{x}$. We next make the crucial paraxial approximation (small angle approximation) corresponding to the situation with

$$
\frac{\partial^{2} A}{\partial z^{2}}<2 i k_{0} \frac{\partial A}{\partial z}
$$

so that we have

$$
2 i k_{0} \frac{\partial A}{\partial z}+\triangle_{\perp} A+\left[\omega^{2} \gamma^{2}(1+\nu)-k_{0}^{2}\right] A=2 i k_{0} \frac{\partial A}{\partial z}+\triangle_{\perp} A+\omega^{2} \gamma^{2} \nu A=0,
$$

for $k_{0}=\omega \gamma$. This approximation requires that we consider wave propagation in a narrow beam geometry, not close to the source and that the medium is weakly inhomogeneous. The resulting equation is called the narrow-angle parabolic equation (PE). In the PE method we take into account only waves traveling in the positive $z$ direction; back scattering is neglected, see $[8,15]$.

3. Decomposition Of Waves In One Dimensional Case . We next aim to extend the parabolic approximation to a strongly range or 'depth' dependent medium and consider first the layered or one-dimensional case. The field is decomposed into outgoing and incoming components. Our equations are based on a decomposition of the field, instead of an operator factorization. The parabolic approximation is a special case of our approach.

In the one dimensional case the bulk modulus $K=K(z)$ depends on $z$ only, the reduced wave equation is now:

$$
p_{z z}+(1+\nu) \omega^{2} \gamma^{2} p=0
$$

with $\gamma=\gamma(z)$. Define

$$
\tau(z)=\int_{z_{s}}^{z} \gamma(s) d s
$$

where $z_{s}$ is the location of source signal. The phase $\tau(z)$ is the travel time from the source to depth $z$ for a plane wave traveling in the depth direction. Equation (7) can then be written as:

$$
p_{z z}+(1+\nu) \omega^{2} \tau_{z}^{2} p=0
$$


Let the pressure $p$ be decomposed as:

$$
p=A(z) e^{i \omega \tau}+B(z) e^{-i \omega \tau} .
$$

Note that by writing the pressure in this way we introduced two degrees of freedom. Therefore, we need an additional constraint on the amplitudes beyond (8).

The velocity is

$$
u=\frac{\gamma}{\rho}\left(A e^{i \omega \tau}-B e^{-i \omega \tau}\right)-\frac{i}{\omega \rho}\left(A_{z} e^{i \omega \tau}+B_{z} e^{-i \omega \tau}\right),
$$

and we make the ansatz

$$
A_{z} e^{i \omega \tau}+B_{z} e^{-i \omega \tau}=0 .
$$

This is the additional constraint on the amplitudes. With it, the velocity becomes:

$$
u=\frac{\gamma}{\rho}\left(A e^{i \omega \tau}-B e^{-i \omega \tau}\right),
$$

and we have decomposed velocity and pressure into down $(A)$ and up-propagating (B) components.

From (9), it follows that

$$
\begin{aligned}
& A_{z}=-B_{z} e^{-2 i \omega \tau} \\
& B_{z}=-A_{z} e^{2 i \omega \tau}
\end{aligned}
$$

and moreover

$$
\left(A_{z z}+i \omega \gamma A_{z}\right) e^{i \omega \tau}+\left(B_{z z}-i \omega \gamma B_{z}\right) e^{-i \omega \tau}=0 .
$$

Combining the above relations and Helmholtz equation (7) we find the following equations for the amplitudes $A$ and $B$ :

$$
\begin{aligned}
& 2 \tau_{z} A_{z}+\tau_{z z} A=i \omega^{2} \tau_{z}^{2} \nu\left(A+B e^{-2 i \omega \tau}\right)+\tau_{z z} B e^{-2 i \omega \tau} \\
& 2 \tau_{z} B_{z}+\tau_{z z} B=-i \omega^{2} \tau_{z}^{2} \nu\left(A e^{2 i \omega \tau}+B\right)+\tau_{z z} A e^{2 i \omega \tau} .
\end{aligned}
$$

We may interpret $A$ and $B$ in the decomposition (8) as amplitudes of the downand up-propagating wave modes respectively.

The decomposition that we introduced above means that we replaced the problem of solving (7) to that of solving the system (10) and (11) and in order to do so we need to introduce boundary conditions for the amplitudes. Note that in the case where the medium is homogeneous, with $\nu \equiv 0$ and $\gamma$ constant, the amplitude equations decouple. The term associated with $A$ then corresponds exactly to the wave component traveling in the positive range or depth direction and the term $B$ with the wave component traveling in the opposite direction. In the general case the wave components couple and correspond to approximate locally up and down traveling wave terms.

In Section 6 we will introduce an iterative based numerical scheme for computing approximate solutions to the system (10) and (11). 
4. Decomposition In Terms Of Generalized Plane Wave Components. We consider next the case with waves propagating in a three dimensional medium with a constant background slowness $\gamma$, but with general three dimensional medium fluctuations modelled by $\nu$. The governing Helmholtz equation is

$$
\triangle p+(1+\nu) \omega^{2} \gamma^{2} p=0
$$

and we now define

$$
\tau(z)=\int_{z_{s}}^{z} \sqrt{\frac{\rho}{K_{p}}-|\kappa|^{2}} d s=\left(z-z_{s}\right) \sqrt{\gamma^{2}-|\kappa|^{2}}, \quad \text { and } \quad S^{ \pm}=\kappa \cdot \mathbf{x} \pm \tau,
$$

with $\kappa$ being the lateral slowness vector. Note that $S^{+}$is a plane wave phase corresponding to waves traveling in the spatial direction $\left(\kappa, \sqrt{\gamma^{2}-|\kappa|^{2}}\right)$. In the case with a general three dimensional background the phase terms $S^{ \pm}$will be solutions of the Eiconal equation associated with the slowness $\gamma(\mathbf{x}, z)$, see [1].

We decompose the wave into up-ward and down-ward modes as above:

$$
\begin{aligned}
& p=A e^{i \omega S^{+}}+B e^{i \omega S^{-}}, \\
& 0=A_{z} e^{i \omega S^{+}}+B_{z} e^{i \omega S^{-}} .
\end{aligned}
$$

In this generalized case the mode coupling transport equations become:

$$
\begin{aligned}
2 \nabla S^{+} \cdot \nabla A+\triangle S^{+} A & -i \omega \gamma^{2} \nu\left(A+B e^{i \omega\left(S^{-}-S^{+}\right)}\right) \\
& =\frac{i}{\omega} \triangle_{\perp} A-R^{-} e^{i \omega\left(S^{-}-S^{+}\right)}, \\
2 \nabla S^{-} \cdot \nabla B+\triangle S^{-} B & -i \omega \gamma^{2} \nu\left(B+A e^{i \omega\left(S^{+}-S^{-}\right)}\right) \\
& =\frac{i}{\omega} \triangle_{\perp} B-R^{+} e^{i \omega\left(S^{+}-S^{-}\right)},
\end{aligned}
$$

with

$$
\begin{aligned}
& R^{+}=2 \nabla_{\perp} S^{+} \cdot \nabla_{\perp} A+\triangle S^{+} A-\frac{i}{\omega} \triangle_{\perp} A, \\
& R^{-}=2 \nabla_{\perp} S^{-} \cdot \nabla_{\perp} B+\triangle S^{-} B-\frac{i}{\omega} \triangle_{\perp} B,
\end{aligned}
$$

where $\triangle_{\perp}$ is the transverse Laplacian. In the case that the fluctuations and the reflected field vanish, $(\nu \equiv 0, B \equiv 0)$, (14) becomes

$$
2 \nabla S^{+} \cdot \nabla A+\triangle S^{+} A=\frac{i}{\omega} \triangle_{\perp} A
$$

which in the high frequency limit gives

$$
2 \nabla S^{+} \cdot \nabla A_{0}+\triangle S^{+} A_{0}=0
$$

that is, the leading order transport equation of geometrical optics.

Here, we will consider the generalization of the parabolic case with waves propagating primarily in the $z$ direction and set $\kappa=0$. Then the coupling transport equations become

$$
\begin{aligned}
2 i k A_{z}+\triangle_{\perp} A+k^{2} \nu A & =-\left(k^{2} \nu B+\triangle_{\perp} B\right) e^{-2 i k z} \\
-2 i k B_{z}+\triangle_{\perp} B+k^{2} \nu B & =-\left(k^{2} \nu A+\triangle_{\perp} A\right) e^{2 i k z},
\end{aligned}
$$


with $k=\gamma \omega$.

In the case that the reflected wave vanish, $B \equiv 0,(16)$ becomes

$$
2 i k A_{z}+\triangle_{\perp} A+k^{2} \nu A=0
$$

which is the standard narrow-angle parabolic approximation. On the other hand, in the case that there is no lateral variation in the amplitudes we find

$$
\begin{aligned}
& 2 i k A_{z}=-k^{2} \nu\left(A+B e^{-2 i k z}\right), \\
& 2 i k B_{z}=k^{2} \nu\left(A e^{2 i k z}+B\right) .
\end{aligned}
$$

These are the transport equations associated with the layered three dimensional case which are analyzed in for instance [1], [11] and [17]. In fact, in this layered case a particular $\kappa$ in the generalized decomposition corresponds to a specific plane wave mode. This variable is then the Fourier variable dual to the lateral space variable $\mathbf{x}$ introduced when the wave field in space and time is transformed into plane wave modes via Fourier transformation with respect to the lateral spatial coordinates.

We next continue our discussion of the system (16) and (17) by introducing specific boundary conditions and a scheme for numerical approximation of the solution.

\section{Boundary Conditions.}

5.1. Initial Condition For Parabolic Equations. We assume that the scatterer $\nu$ is compactly supported and is located in a slab of thickness $L$, so that $k$ is constant for $z<0$ and $z>L$. The source is located in the homogeneous medium, at $z_{s}<0$. Recall that with $B=0$ the amplitude equation for $A$ is equivalent to the standard parabolic equation. We impose an initial condition for the down-ward field $A$ at $z=0$ and for the reflected field $B$ at $z=L$. At the top, we use a Gaussian form for the initial data [19]:

$$
A(\mathbf{x}, 0)=\sqrt{k_{0}} e^{-\frac{|\mathbf{x}|^{2}}{2}}
$$

Since the medium is homogeneous for $z>L$ and there are no sources located in this half space we set

$$
B(\mathbf{x}, L)=0
$$

5.2. Artificial Boundary. The scatterer $\nu$ is located in infinite homogeneous medium. We truncate the domain by adding PML (Perfectly Matched Layer) artificial boundary in the lateral dimensions. The idea is to introduce an exterior layer at the artificial boundary in such a way that all plane waves are totally absorbed, and no reflection occurs at the boundary [2]. For simplicity, we now assume two spatial dimensions corresponding to one lateral dimension. In order to introduce the artificial boundary condition we return to the Helmholtz equation:

$$
p_{z z}+p_{x x}+k^{2}(1+\nu(x, z)) p=0 .
$$

In the matched layer we change this equation to obtain damping of the plane wave modes. We introduce the governing equations 


$$
\frac{\partial^{2} p}{\partial z^{2}}+\frac{i \omega}{\sigma-i \omega} \frac{\partial}{\partial x}\left(\frac{i \omega}{\sigma-i \omega} \frac{\partial p}{\partial x}\right)+k^{2}(1+\nu) p=0,
$$

where $\sigma(x)>0$ in the artificial domain giving damping of the plane wave modes, whereas $\sigma(x)=0$ in the physical domain giving the Helmholtz equation there. Denote

$$
s(x)=\frac{i \omega}{\sigma(x)-i \omega},
$$

then with the PML, the coupling transport equations can be written

$$
\begin{aligned}
2 i k A_{z}+ & s^{2}(x) A_{x x}+k^{2} \nu A+s(x) s^{\prime}(x) A_{x} \\
& =-\left(k^{2} \nu B+s^{2}(x) B_{x x}+s(x) s^{\prime}(x) B_{x}\right) e^{-2 i k z} \\
-2 i k B_{z} & +s^{2}(x) B_{x x}+k^{2} \nu B+s(x) s^{\prime}(x) B_{x} \\
& =-\left(k^{2} \nu A+s^{2}(x) A_{x x}+s(x) s^{\prime}(x) A_{x}\right) e^{2 i k z}
\end{aligned}
$$

The ideal case of using these two coupled parabolic equations for the forward wave field $A$ and backward wave field $B$ in numerical computations would be that we can solve them in the form of Jacobi iteration, i.e., solve $A$ with current $B$ and then solve $B$ with current $A$ iteratively. This would reduce the computation of the Helmholtz equation into the computation of a sequence of parabolic equations. However, as we will show in the appendix, this iterative procedure is not stable. Since we are interested in the narrow angle wave propagation in the $z$-direction, we drop the lateral scattering terms $\triangle_{\perp} B$ ( or $\triangle_{\perp} A$ ) when we solve for $A(x, z)$ ( or $B(x, z)$ ) in (16) (or (17)) which cause the instability. Consider the equation (16). In the homogeneous case with $\nu \equiv 0$ the terms involving the reflected field $B$ will be lower order correction terms to the paraxial approximation. We will consider regimes where there is significant backscattering due to the scatterer $\nu$ and therefore retain the term involving $\nu$ in the coupling part of equations (16) and (17). With PML included we have the following two coupled parabolic approximations (with one lateral dimension):

$$
\begin{aligned}
& 2 i k A_{z}+s^{2}(x) A_{x x}+k^{2} \nu A+s(x) s^{\prime}(x) A_{x}=-k^{2} \nu B e^{-2 i k z}, \\
& -2 i k B_{z}+s^{2}(x) B_{x x}+k^{2} \nu B+s(x) s^{\prime}(x) B_{x}=-k^{2} \nu A e^{2 i k z}, \\
& A(x, 0)=\sqrt{k_{0}} e^{-\frac{|x|^{2}}{2}} ; \quad B(x, L)=0 .
\end{aligned}
$$

We next introduce a numerical scheme for approximation of the solution to this system.

6. Numerical Solution. We will solve the system $(20,21)$ by iteration. In the first step we compute an approximation for $A$ using (20) with $B=0$. Next, we solve for $B$ using (21) with the computed approximation for $A$. This procedure is then repeated with the updated values for $A$ and $B$. Observe that in the homogeneous case with $\nu=0$ the iteration converges after the first step with $B \equiv 0$ and $A$ solving the standard narrow-angle parabolic equation.

We now discretize the above iteration by introducing finite differences for the derivatives. The discretized field variables are denoted $A_{l}^{m}$ and $B_{l}^{m}$, where $l$ and $m$ are grid indices in $x$ and $z$, respectively. Assuming the field $A$ is known at step $m$, we 
proceed to determine the field at step $m+1$. For $B$, we do it backward, i.e., determine the field at step $m$ from the $m+1$ step.

We use Crank-Nicolson scheme to solve equation (20). At the half grid point $\left(x_{l}, z_{m+\frac{1}{2}}\right)$, we have

$$
\begin{aligned}
& 2 i k A_{z}\left(x_{l}, z_{m+\frac{1}{2}}\right)+k^{2} \nu A\left(x_{l}, z_{m+\frac{1}{2}}\right)+s^{2}\left(x_{l}\right) A_{x x}\left(x_{l}, z_{m+\frac{1}{2}}\right) \\
& +s\left(x_{l}\right) s^{\prime}\left(x_{l}\right) A_{x}\left(x_{l}, z_{m+\frac{1}{2}}\right)=-k^{2} \nu B\left(x_{l}, z_{m+\frac{1}{2}}\right) e^{-2 i k z_{m+\frac{1}{2}}} .
\end{aligned}
$$

By using the standard finite difference formulae, we can express the solution in vector form as

$$
\begin{aligned}
& {\left[u_{l}, v_{l}^{m+\frac{1}{2}}, w_{l}\right]\left[\begin{array}{c}
A_{l-1}^{m+1} \\
A_{l}^{m+1} \\
A_{l+1}^{m+1}
\end{array}\right] } \\
= & {\left[-u_{l}, \hat{v}_{l}^{m+\frac{1}{2}},-w_{l}\right]\left[\begin{array}{c}
A_{l-1}^{m} \\
A_{l}^{m} \\
A_{l+1}^{m}
\end{array}\right]-2 k^{2} h^{2} \nu_{l}^{m+\frac{1}{2}}\left(B_{l}^{m+1}+B_{l}^{m}\right) e^{-2 i k z_{m+\frac{1}{2}}}, }
\end{aligned}
$$

where

$$
\begin{aligned}
u_{l} & =\left(2 s^{2}\left(x_{l}\right)-s\left(x_{l}\right) s^{\prime}\left(x_{l}\right) h\right), \\
v_{l}^{m+1 / 2} & =8 i k \frac{h^{2}}{\tau}+2 k^{2} h^{2} \nu_{l}^{m+\frac{1}{2}}-4 s^{2}\left(x_{l}\right), \\
w_{l} & =\left(2 s^{2}\left(x_{l}\right)+s\left(x_{l}\right) s^{\prime}\left(x_{l}\right) h\right), \\
\hat{v}_{l}^{m+1 / 2} & =8 i k \frac{h^{2}}{\tau}-2 k^{2} h^{2} \nu_{l}^{m+\frac{1}{2}}+4 s^{2}\left(x_{l}\right) .
\end{aligned}
$$

To complete the formulation we finally collect the local solutions given by (23) into a global matrix solution over the mesh points in depth.

The coefficient of $B_{z}$ in (21) is negative of that of $A_{z}$ in (20), however, since we solve $B$ from bottom to top, we will determine $B$ at level $m$ from level $m+1$. Then, we obtain the same matrix formulation as when solving for $A$ (modulo the sign of the phase):

6.1. Numerical Example. We test our algorithm by considering a sound signal propagating through a random medium with some imbedded compact scatterers.

There are 20 imbedded strong scatterers in the domain, they are strongly anisotropic with dimensions about $50 \mathrm{~m}$ in the vertical direction and $150 \mathrm{~m}$ in the horizontal dimension. The signal speed in the homogeneous medium is $330 \mathrm{~m} / \mathrm{s}$ and the wavelength is about $13 \mathrm{~m}$ and the speed is increased by $50 \%$ in the scatterers. In addition there are random fluctuations in the refractive index of relative magnitude $1 \%$ and correlation length $10 \mathrm{~m}$.

Our computational domain contains 20 wavelength (horizontal) and 200 wavelength (vertical). We use 10 grid points per wavelength in both directions, whereas on the left and the right sides, we use 25 grid points for the PML medium. The medium and the computational domain and the Helmholtz solution are shown in Figure 1 (a) and (b).

After 15 iterations, we get the solution for the coupled parabolic equations. The solutions of Parabolic approximation and Coupled Parabolic approximation are shown 


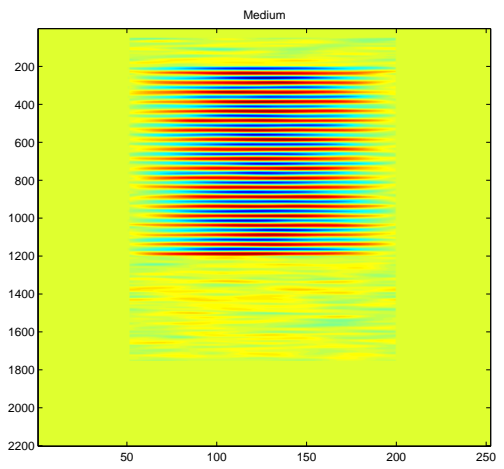

(a) Medium

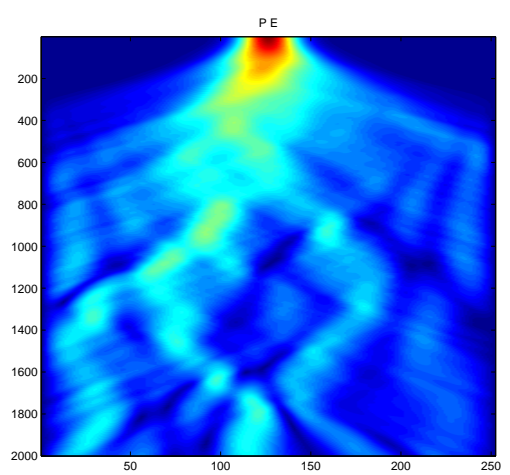

(c) PE Solution

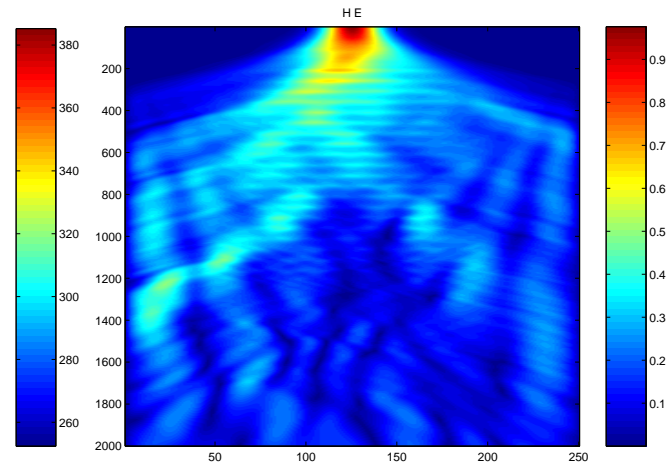

(b) HE Solution

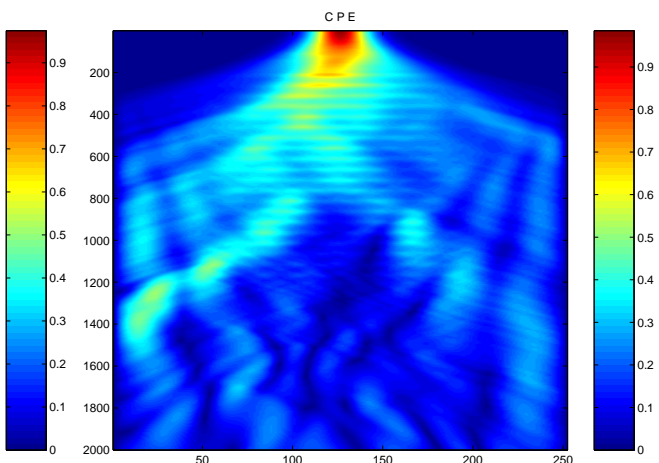

(d) C-PE Solution

FIG. 1. Medium and Solutions

in Figures 1 (c), (d) respectively and we see that the Coupled Parabolic approximation captures well the spatial structures of the Hemholtz solution.

In Figure 2 we show $p$ at the center of the domain along the propagation direction in $z$. The solid line is the numerical solution of the Helmholtz equation. The solution of the parabolic equation is shown by the dashed line in the left plot. The solution of the coupled parabolic equations (C-PE) using the algorithm described in the previous section is shown by the dashed line in the right plot. Note that our C-PE solution is very close to the Helmholtz solution whereas the PE solution fails to capture the oscillations in $p$ that is due to the imbedded scatterers which give a significant backscattering.

Figure 3 are the spectrum of the signal in the center of the domain solved by the different methods. Here we compute the spectrum as the Fourier transform of the squared magnitude of the signal. The solid line is the spectrum associated with the Helmholtz solver, while the dashed lines are the spectra for PE (a) and C-PE (b) respectively. The spectra associated with the Helmholtz and coupled PE solvers are indeed very similar.

7. conclusion. In this paper we develop an algorithm using two coupled parabolic equations to simulate wave propagation over long distances. The two coupled parabolic equations are derived from a wave field decomposition. Both forward and backward propagation field components, as well as their interactions, are captured. Though not solving the Helmholtz equation, our method can deal with strong inho- 


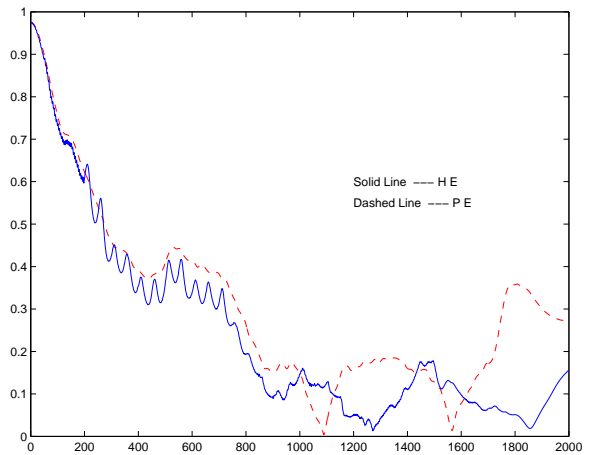

(a) Helmholtz and PE

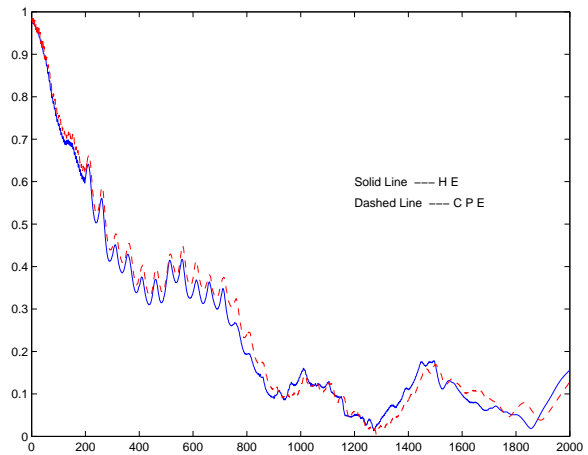

(b) Helmholtz and C-PE

FIG. 2. The solutions computed by different equations.

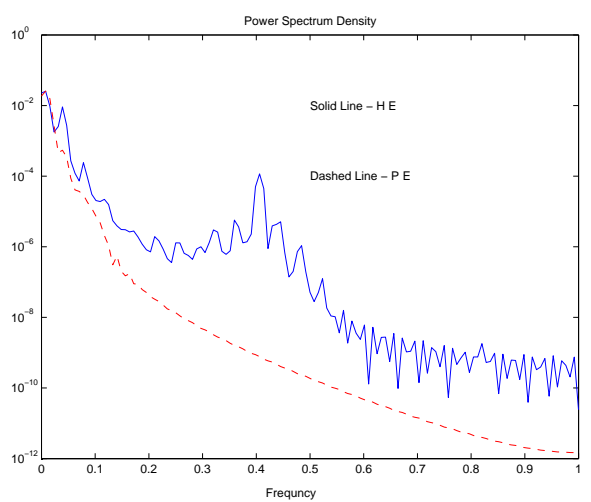

(a) Helmholtz and PE

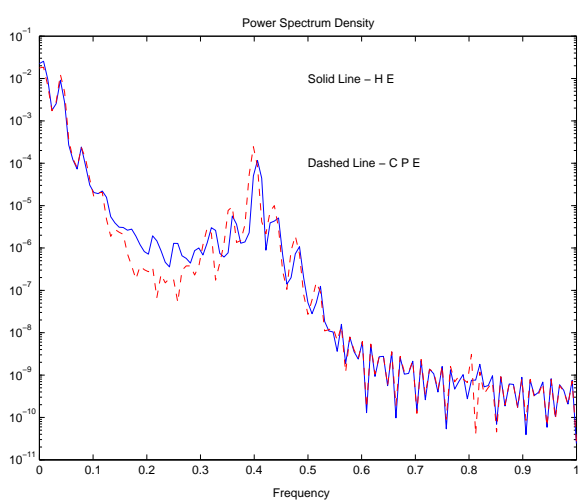

(b) Helmholtz and C-PE

FIG. 3. The Spectrum computed by different algorithms

mogeneities with arbitrary geometry in the medium and the computational cost is comparable to that of the parabolic approximation. A main objective has been to capture the structure of the high frequency components of the solution caused by rapid incoherent fluctuations in the medium parameters and this has been accomplished in our numerical example.

Appendix. In this section, we show that the system $(18,19)$ is unstable using Fourier analysis. We consider the system in the homogeneous case with $\gamma=0$ :

$$
\begin{aligned}
2 i k A_{z}+A_{x x}+B_{x x} e^{-2 i k z} & =0, \\
-2 i k B_{z}+B_{x x}+A_{x x} e^{2 i k z} & =0 .
\end{aligned}
$$

We are looking for the solution in the form:

$$
A(x, z)=a(z) e^{2 i \pi n x} \text { and } B(x, z)=b(z) e^{2 i \pi n x} .
$$

In the first iteration, we set $B=0$ in $(24)$, the equation then becomes

$$
2 i k A_{z}+A_{x x}=0
$$


and we are looking for a solution of the type $A(x, z)=a(z) e^{2 i \pi n x}$ with initial condition $a(0)=1$. The solution is given by

$$
a(z)=e^{-4 i \pi^{2} n^{2} z /(2 k)} .
$$

Next, with the above $A(x, z)$, we solve for $B=b(z) e^{2 i \pi n x}$ in the equation (25) with the prescribed initial value at the bottom $b(1)=0$, the solution is then

$$
b(z)=\frac{\left(-4 \pi^{2} n^{2}\right)\left[e^{i z\left(4 k^{2}-4 n^{2} \pi^{2}\right) /(2 k)}-e^{i\left(-8 n^{2} \pi^{2}+4 k^{2}+4 n^{2} \pi^{2} z\right) /(2 k)}\right]}{-4 k^{2}+8 n^{2} \pi^{2}} .
$$

In the 2nd iteration, we solve for $A$ with the $B(x, z)$ from the 1st iteration, and solve for $B$ with the obtained $A$, we find then

$$
|a(z)|=O\left(n^{2}\right) \text { and }|b(z)|=O\left(n^{4}\right) .
$$

In 3rd iteration, we will have

$$
|a(z)|=O\left(n^{6}\right) \text { and }|b(z)|=O\left(n^{8}\right) .
$$

The order of $n$ in the amplitude will in general increase by two in each iteration and we see that this iteration scheme for the system $(24,25)$ is unstable.

Since we are interested in wave propagation in the $z$-direction, we drop the term $B_{x x}$ (respectively $A_{x x}$ ) when we solve for $A(x, z)$ (respectively $B(x, z)$ ) to obtain the system

$$
\begin{gathered}
2 i k A_{z}+k^{2} \gamma A(x, z)+A_{x x}+k^{2} \gamma B(x, z) e^{-2 i k z}=0, \\
-2 i k B_{z}+k^{2} \gamma B(x, z)+B_{x x}+k^{2} \gamma A(x, z) e^{2 i k z}=0 .
\end{gathered}
$$

If the same analysis were applied to the above system, we would find that the order of amplitudes of $A(x, z)$ and $B(x, z)$ remain the same during the iteration.

\section{REFERENCES}

[1] M. Ash, W.Kohler, G.C. Papanicolaou, M. Postel and B. White, Frequency content of randomly scattered signals, SIAM Review, 33 (1991), pp. 519-625.

[2] Jean-Pierre Berenger, A perfectly matched layer for the absorption of electromagnetic waves, Journal of computational physics, 114 (1994), pp. 185-200.

[3] Francis Collino, Souad Ghanemi and Patrick Joly, Domain decomposition method for harmonic wave propagation: a general presentation, Comput. Methods Appl. Mech. Engrg., 184 (2000), pp. 171-211.

[4] Michael D. Collins and Richard B. Evans, A two-way parabolic equation for acoustic backscattering in the ocean, J. Acoust. Soc. Am., 91:3 (1992), pp. 1357-1368.

[5] M. V. DE Hoop AND A. K. Gautesen, Uniform asymptotic expansion of the square-root Helmholtz operator and the one-way wave propagator, SIAM J. Appl. Math., 63:3 (2003), pp. $777-800$.

[6] Ding Lee, William L. Siegman and Donald F. St. Mary, A numerical marching scheme to compute scattering in the ocean, Mathematics and Computers in Simulation, 34 (1992), pp. $525-540$.

[7] Ding Lee; Allan D. Pierce and Er-Chang Shang, Parabolic Equation Development in the Twentieth Century, Journal of Computational Acoustics, 8:4 (2000), pp. 527-637.

[8] Finn B. Jensen, Willian A. Kuperman, Michael B. Porter and Henrik Schmidt, Computational Ocean Acoustics, AIP Press, 2nd ed. 2000.

[9] L. Fishman, One-way wave propatation methods in direct and inverse scalar wave propagation modeling, Radio Science, 28:5 (1993). 
[10] L. Fishman, A.K. Gautesen And Z. Sun, Uniform high-frequency approximations of the square root Helmholtz operator symbol, Wave Motion, 26 (1997), pp. 127-161.

[11] J. F. Clouet and J. P. Fouque, Spreading of a pulse traveling in random media, Ann. Appl. Probab., 4 (1994), pp. 1083-1097.

[12] Y. Lu And J. McLaughlin, The Riccati method for the Helmholtz equation, J. Acoust. Soc. Am., 100:3 (1996).

[13] Y. Lu, One-way Large Range Step Methods for Helmholtz Waveguides, Journal of Computational Physics, 152 (1999), pp. 231-250.

[14] Y. Lu, Exact one-way methods for acoustic waveguides, Mathematics and computers in Simulation, 50 (1999), pp. 377-391.

[15] Erik M. Salomons, Computational atmospheric acoustics, Kluwer Academic Publishers, 2001.

[16] K. Solna, Stable Spreading of Acoustic Pulses due to laminated microstructure, Dissertation, Stanford University, 1997.

[17] K. Solna and G. C. Papanicolaou, Ray Theory for a Locally Layered Medium, Waves in Random Media, 10 (2000), pp. 151-198.

[18] M. J. N. van Straalen, M. V. De Hoop and H. Blok, Generalized Bremmer series with rational approximation for the scattering of waves in inhomogeneous media, 104:4 (1998), pp. 1943-1963.

[19] F.D. TAPPERT, The parabolic approximation method, in Wave Progpagation in Underwater Acoustics, edited by J.B. Keller and J.S. Papadakis (Springer-Verlag, NewYork, 1977), pp. $224-287$. 
